\title{
ECTODERMAL DYSPLASIA- A CASE OF X-LINKED RECESSIVE DISORDER
}

\author{
Avinash Laxmanrao Kashid ${ }^{1}$, Atulkumar Ashokrao Patil ${ }^{2}$
}

${ }^{1}$ Assistant Professor, Department of Dentistry, Swami Ramanand Teerth Rural Government Medical College and Hospital, Ambajogai, Maharashtra.

2Senior Resident, Department of Dentistry, Swami Ramanand Teerth Rural Government Medical College and Hospital, Ambajogai, Maharashtra.

HOW TO CITE THIS ARTICLE: Kashid AL, Patil AA. Ectodermal dysplasia- a case of $\mathrm{x}$-linked recessive disorder. J. Evolution Med. Dent. Sci. 2018;7(13):1673-1676, DOI: 10.14260/jemds/2018/377

PRESENTATION OF CASE

A 12-year-old boy visited the Department of Dentistry at SRTR GMC, Ambajogai with chief complaint of lack of aesthetics and difficulty in mastication. According to familial history, a grandfather of the patient also suffered from oligodontia, hypoplastic midface and heat intolerance (Figure No. 1 and 2).

Ectodermal dysplasia (ED) is a large group of heterogeneous heritable conditions characterised by congenital defects of two or more ectodermal structures and their appendages: hair, nails, teeth and sweat glands. ${ }^{1}$

Thurnam published the first report of a patient with ectodermal dysplasia in $1848 .^{2}$ There are 2 major types of this condition depending on the number and functionality of the sweat glands: (1) X-linked anhidrotic or hypohidrotic, (ChristSiemens-Touraine syndrome) and (2) Hidrotic, (Clouston's syndrome). ${ }^{3}$

ED syndrome cases usually show manifestations of typical general mental development, frontal bossing with characteristics reduction in amount of hair, absence of sweat glands resulting in temperature elevation, absence of sebaceous glands resulting in dry skin, depressed nasal bridge, protuberant lips, prominent supraorbital ridges, sunken cheeks, wrinkled hyperpigmented skin around the eyes and large low-set ears.

Oral traits of ectodermal dysplasia (ED) may be expressed as anodontia or hypodontia with or without a cleft lip and palate. Other characteristic oral findings include the reduced vertical dimension of the lower face, disappearance of the vermilion border, malformed teeth, the oral mucosa becomes dry, xerostomia and the lips become prominent. 4

The most common prosthetic treatment for the dental management of ectodermal dysplasia is removable prosthodontics. Restoring function and appearance of children with ectodermal dysplasia is more challenging than usual due to presence of little or no bone ridge. In this case report, it is aimed to describe the prosthetic rehabilitation of young boy with anhidrotic ectodermal dysplasia associated with severe anodontia.

\section{CLINICAL DIAGNOSIS}

On general examination the patient presented with the classical triad of hypohidrosis, hypotrichosis and hypodontia. On clinical examination, dry and scaly skin with slight

'Financial or Other Competing Interest': None.

Submission 30-01-2018, Peer Review 10-03-2018,

Acceptance 17-03-2018, Published 26-03-2018.

Corresponding Author:

Dr. Atulkumar Ashokrao Patil,

Rutuja Niwas, Pise Colony, Gargoti Tq.,

Bhudargad District, Kolhapur-416209.

E-mail: atulkumarpatil85@gmail.com

DOI: $10.14260 /$ jemds $/ 2018 / 377$

\section{(c) $($ ) $९$}

elevation of temperature was observed. Hair examination revealed fine sparse hair, lustreless appearance and very thin eyebrows.

Eye examination shows dryness of cornea and decreased lubrication. Finger examination revealed normal shaped fingers with thin nails. Ear examination revealed large low-set ears with midface hypoplasia gives him an older look.

Apart from this the patient presented with depressed nasal bridge, protuberant lips, frontal bossing and sunken cheeks with prominent supraorbital ridges (Figure 3 and 4). The intraoral examination revealed complete edentulism in the lower jaw. Four peg-shaped anterior teeth and four molars with extra cusps were present in the upper jaw (Figure No. 5 and 6). The ridges were atrophic except the region where the teeth were present.

Reduced vertical bone heights of both the arches were of considerable significance. Oral panoramic radiograph confirmed the absence of other permanent tooth buds. It also revealed taurodontism with molar teeth in the maxillary arch and underdeveloped mandibular ridge (Figure No. 7). So with the above clinical and radiological findings, a diagnosis of Hypohidrotic Ectodermal Dysplasia was made.

\section{MANAGEMENT}

Treatment plan included making complete denture for the lower jaw to improve appearance, function and speech. Preliminary impression was made by impression compound, then custom tray was prepared for final impression.

Final impression was made by using green stick compound and zinc oxide eugenol pastes. Jaw relationship was recorded using temporary base and wax rim. The arranged teeth were verified in the mouth during the try-in appointment. After laboratory processing, the denture was delivered to the patient with proper instructions. Follow-ups of every six months were planned for adjustment or replacement of older denture and orthodontic treatment for upper teeth.

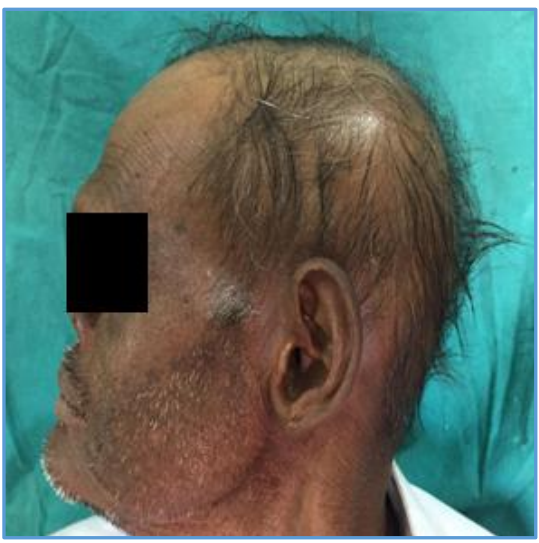

Figure No. 1 Profile View of Patient's Maternal Grandfather 


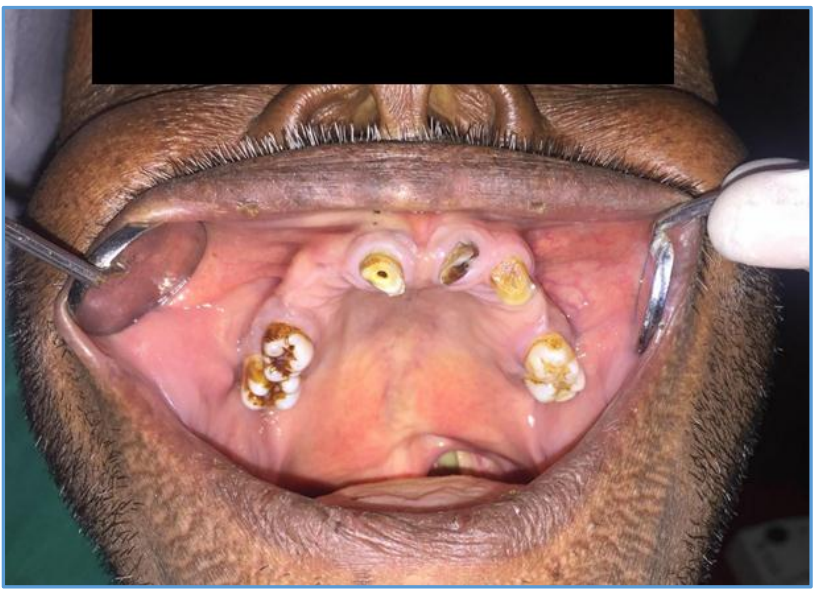

Figure No. 2 Intra-oral View of Patient's Maternal Grandfather

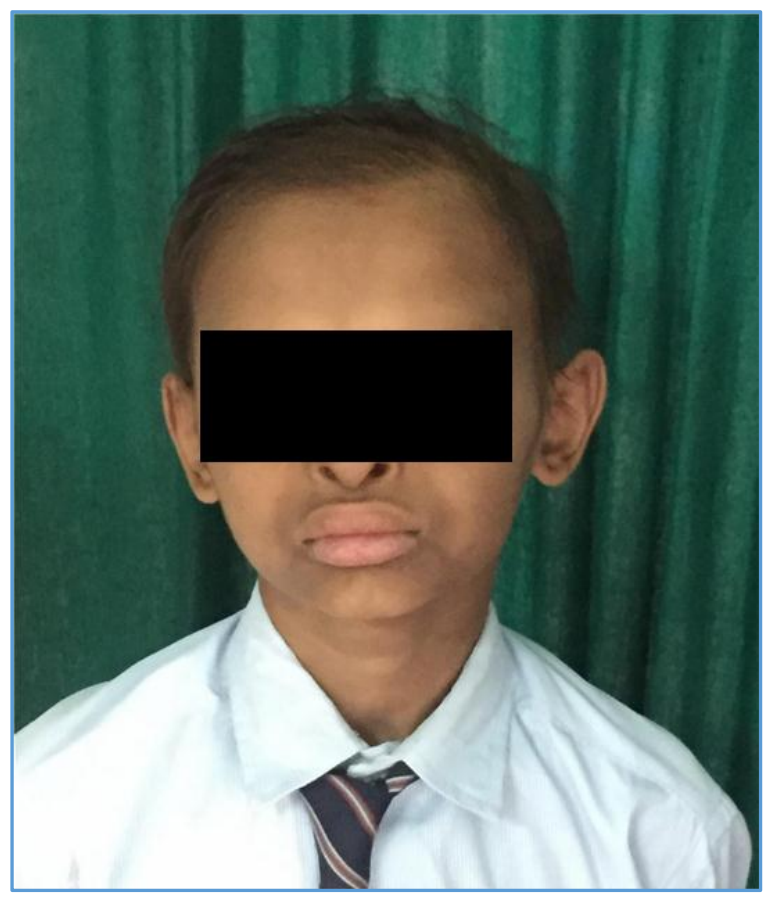

Figure No. 3 Facial View of the Patient

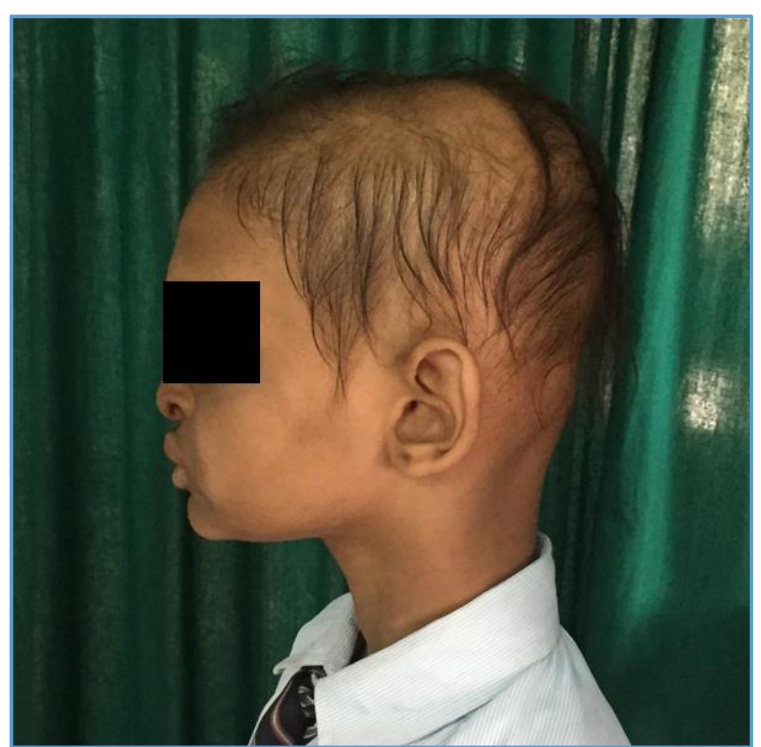

Figure No. 4 Profile View of the Patient

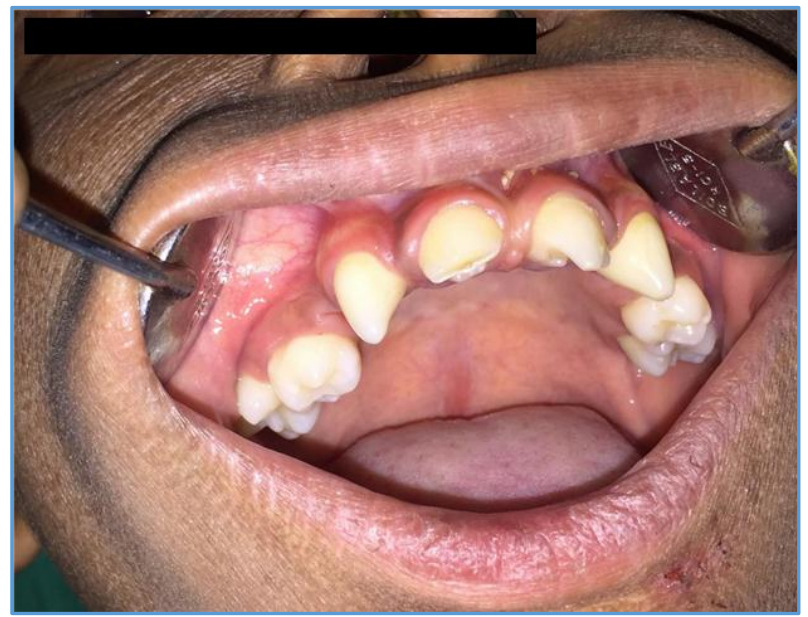

Figure No. 5 Intra-oral View of Upper Jaw

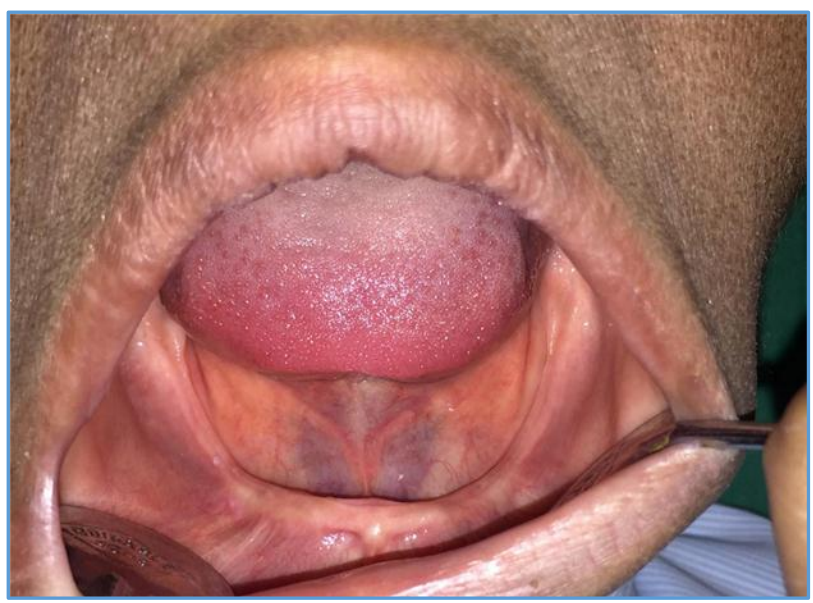

Figure No. 6 Intra-oral View of Lower Jaw

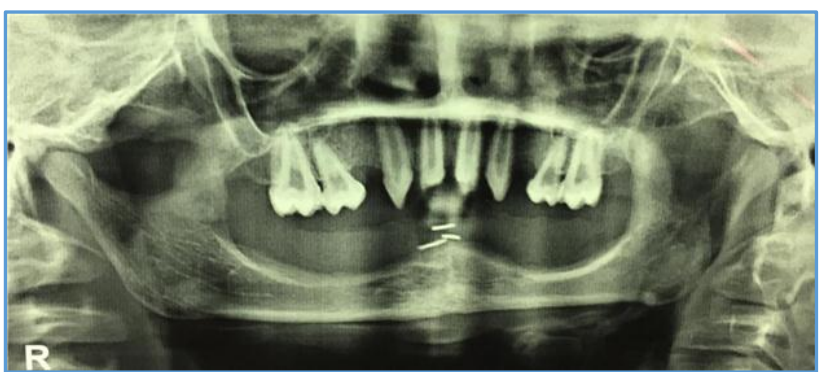

Figure No. 7 Panoramic Radiograph of Patient

\section{DISCUSSION}

Ectodermal dysplasia (ED) is a large group of heterogeneous heritable conditions characterised by congenital defects of two or more ectodermal structures and their appendages: hair (hypotrichosis, partial or total alopecia), nails (dystrophic, hypertrophic, abnormally keratinised), teeth (enamel defect or absent) and sweat glands (hypoplastic or aplastic).

Thurnam published the first report of a patient with ectodermal dysplasia in 1848.1,5 The term ectodermal dysplasia was not coined until 1929. In 1971, only eight forms of ED were known. Now approximately 200 different ED have been delineated; about 30 have been identified at the molecular level with identification of the causative gene. 6 
There are several classifications given by different authors. Some are based on clinical features and others on genetic component of the disorder. Clinically, there are two major types of ED, namely hidrotic and anhidrotic (hypohidrotic form). Lamartine classified the ED according to the function of their mutated genes into 4 functional groups: cell-cell communication and signaling; adhesion; transcription regulation; and development. ${ }^{7}$ Recently, a new classification for ED has been proposed, based on the molecular genetic data by Priolo who divided the ED into two groups. The first group includes disorder in which a defect in the epithelial-mesenchymal interaction and the second group involved defect in cell-cell adhesion and communication. ${ }^{8}$

The EDA, EDAR and EDARADD genes provide instructions for making proteins that work together during embryonic development.

These proteins form part of a signaling pathway that is critical for the interaction between two cell layers, the ectoderm and the mesoderm. It is essential for the formation of several structures that arises from ectoderm including the skin, hair, nails, teeth and sweat glands.

Mutation in these genes prevents normal interaction between the ectoderm and mesoderm and impairs the normal development of hair, sweat glands and the teeth. The improper formation of these ectodermal structures leads to the characteristic features of HED. ${ }^{9}$

Hypohidrotic (or anhidrotic) ectodermal dysplasia (HED) is the most frequent form of ED that can be inherited in an Xlinked $(\mathrm{XL})$, autosomal recessive or autosomal dominant manner. A novel signaling molecule of the tumour necrosis factor (TNF) superfamily named ectodysplasin (EDA) was responsible for X-linked form of HED. ${ }^{10}$

Autosomal forms of HED are due to mutation in the EDA receptor (EDAR) and EDAR associated death domain (EDARADD).4,11 The present case was diagnosed as X-linked form of HED due to presence of same clinical feature in maternal grandfather. Both the patients and his maternal grandfather were referred to higher genetic centre for studying and better understanding of genetic pathway.

Hypohidrosis is possibly the most remarkable characteristic of ED, because it may not be apparent in the first year of life but present later as a fever of unknown origin. The inability to sweat results in intolerance to heat, occasionally hyperpyrexia or high fevers, which further leads to seizures and other neurological sequelae.

This is one of the most common causes of mortality with a $30 \%$ rate in infancy and early childhood. ${ }^{4}$ In the present case, the patient was diagnosed with unknown cause of hyperpyrexia at age of 6 months and was treated successfully.

Other problems associated are pharyngitis, rhinitis, cheilitis and dysphagia may result from decreased number of mucous glands in the respiratory and gastrointestinal tracts. Patient in the present case had problems such as stomatitis and cheilitis, which were relieved by taking symptomatic treatment. In some cases decreased function of certain components of the immune system (depressed lymphocyte function, cellular immune function), potentially causing an increased susceptibility to certain infections. ${ }^{4,12}$

However, patient in present case had good immune system reported till date. The skin covering majority of the body may be abnormally thin, dry and soft. Scalp hair, eyebrows, eyelashes and other body hair may be sparse, poorly developed or absent. There may be hyperkeratosis of the palms of the hands and soles of the feet.

Fingernails and toenails also may show faulty development. Other reported clinical manifestations are frontal bossing; sunken cheeks, prominent supraorbital ridges, a saddle nasal bridge and large low set ears. In addition, the midface is depressed and hypoplastic; the cheek bones are high and broad (malar hypoplasia) and appear flat and depressed, thick everted lips and/or a large chin. The facial appearance of affected individuals is so characteristic of the condition that unrelated patient may even be mistaken for a sibling. ${ }^{4}$ All characteristic findings of ED were found in the reported case.

The most striking oral feature of ED may range from hypodontia to anodontia of the primary or permanent teeth (with or without cleft lip and cleft palate) associated with hypoplasia of the alveolar bone structure.

Consequently, the vertical dimension of the lower face is reduced. The vermilion border disappears resulting in protuberance of lips. ${ }^{13}$ It is not uncommon for the face of an affected child taking appearance characteristics of old age. The average number of missing permanent tooth is reported as 23.7.4,14

The maxillary central incisors, maxillary first molars and maxillary canines are teeth most often present. If teeth are present they are often conical in shape, malformed and widely spaced. Similar findings were noticed in the present case with additional findings of taurodontism and extra cusps present in molar teeth. ${ }^{4}$ The cases of true anodontia were extremely limited to rare conditions.

Tanner states in case of ED such abnormal appearance may affect normal social and psychological development in young patients. 15 Therefore, dental care for a patient with anodontia is extremely important. In such cases, multidisciplinary team is generally advocated to be the most appropriate approach. Treatment decisions of the dental team depend on the patient's needs, wishes, their willingness to undergo minor or major treatment with different impact and also on the economic possibilities.

An early age intervention helps to modify the intraoral prosthesis during growth spurts or rapid growth periods. Prosthetic intervention can be done with a child as young as 2 or 3 years if the child is cooperative.

This also allows the child to adjust with the prosthesis or appliance and develop normal appearance, speech, mastication and swallowing as well as temporomandibular joint function. Apart from dental benefits, an early age intervention also provides psychosocial benefits with some measure of confidence to child. Treatment generally includes a removable and/or fixed partial denture, an overdenture, complete denture prosthesis or an implant retained prosthesis. 16

In cases where there is associated cleft lip and palate, the treatment may consist of intervention by a plastic surgeon and an oral and maxillofacial surgeon. In such cases, a maxillofacial prosthesis may be indicated. ${ }^{16}$ In the present case, prosthodontic management was done by complete denture in lower arch.

The option of removable partial denture was ruled out in upper arch due to insufficient space and could be considered only after orthodontic treatment of remaining teeth. Fixed prosthodontic management is seldom used for such type of 
patients because of minimal number of teeth present and often patients are young, in which fixed partial denture with rigid connector will hamper active growth of dental arches. ${ }^{4,16}$

Options which could be considered in our patient were of overdenture of upper arch after intentional root canal treatment of the existing teeth. But considering the increased number of appointments and disinterest of patient, this option was also ruled out.

Implant placement for an adult patient with ectodermal dysplasia is the treatment of choice. ${ }^{16}$ However, this was not recommended in the present case because of the growing age of the patient and inadequate bone support. The treatment option preferred at present was of a complete denture for lower arch considering his present age.

As the child matures the removable prosthesis needs relining, rebasing or remaking to accommodate growth changes and maintain normal oral functions. When child reaches growth phase, orthodontic treatment may be indicated as better management of spacing and may prepare the mouth for a fixed partial denture or implants in future. ${ }^{4,16}$

Moreover, considering the growth potential of our patient and erupted tooth remained as potential abutment, a treatment option of overdenture, fixed partial denture or implants can be considered in future.

\section{FINAL DIAGNOSIS}

The final diagnosis of the case was X-linked hypohidrotic ectodermal dysplasia.

\section{REFERENCES}

[1] Itin PH. Rationale and background as basis for a new classification of the ectodermal dysplasia. Am J Med Genet A 2009;149A(9):1973-6.

[2] Thurnam J. Two cases in which the skin, hair and teeth were very imperfectely developed. Med Chir Trans 1848;31:71-82.

[3] Nunn JH, Carter NE, Gillgrass TJ, et al. The interdisciplinary management of hypodontia: background and role of paediatric dentistry. Br Dent J 2003;194(5):245-51.

[4] Mehra GRM. Prosthodontic management of children with ectodermal dysplasia: review of literature. Dentistry 2015;5(11):340.
[5] Pinheiro M, Freire-Maia N. Ectodermal dysplasias: a clinical classification and a causal review. Am J Med Genet 1994;53(2):153-62.

[6] Freire-Maia N, Lisboa-Costa T, Pagnan NA. Ectodermal dysplasias: how many? Am J Med Genet 2001;104(1): 84.

[7] Lamartine J. Towards a new classification of ectodermal dysplasias. Clin Exp Dermatol 2003;28(4): 351-5.

[8] Priolo M. Ectodermal dysplasias: an overview and update of clinical and molecular functional mechanisms. Am J Med Genet A 2009;149A(9):200313.

[9] Priolo M, Lagana C. Ectodermal dysplasias: a new clinical-genetic classification. J Med Genet 2001;38(9):579-85.

[10] Kere J, Srivastava AK, Montonem O, et al. X-linked anhidrotic (hypohidrotic) ectodermal dysplasia is caused by mutation in a novel transmembrane protein. Nat Genet 1996;13(4):409-16.

[11] Mikkola ML. Molecular aspect of hypohidrotic ectodermal dysplasia. Am J Med Genet A 2009;149A(9): 2031-6.

[12] Vieira KA, Teixeira MS, Guirado CG, et al. Prosthodontic treatment of hypohidrotic ectodermal dysplasia with complete anodontia: case report. Quintessence Int 2007;38(1):75-80.

[13] Bani M, Tezkirecioglu AM, Akal N, et al. Ectodermal dysplasia with Anodontia: a report of two cases. Eur J Dent 2010;4(2):215-22.

[14] Suri S, Carmichael RP, Tompson BD. Simultaneous functional and fixed appliance therapy for growth modification and dental alignment prior to prosthetic habilitation in hypohidrotic ectodermal dysplasia: a clinical report. J Prosthet Dent 2004;92(5):428-33.

[15] Tanner BA. Psychological aspect of hypohidrotic ectodermal dysplasia. Birth Defects Orig Artic Ser 1988;24(2):263-75.

[16] Murthy JV, Vaze R. Prosthetic management of an ectodermal dysplasia: a case report. People's Journal of Scientific Research 2010;3(2):37-40. 